\title{
Myosin 5 evolution and expression in teleosts
}

\author{
Richard J Nuckels*, Dana M Garcia \\ From Epigenetics \& Chromatin: Interactions and processes \\ Boston, MA, USA. 11-13 March 2013
}

\section{Background}

Myosins make up a superfamily of motor proteins best known for their role in actin-based motility. Myosin 5 (myo5) is an unconventional myosin that shuttles organelles along cytoplasmic actin tracks. Recently, new roles have been ascribed to myosin $5 \mathrm{a}$ and myosin $5 \mathrm{~b}$ - nuclear roles relating to splicing and transcriptional processing, respectively. Vertebrates have undergone two rounds of whole genome duplication and teleost fishes have undergone an additional round of genome duplication. Consequently myo5 exists in numerous forms in vertebrates (myo5a, myo5b, and myo5c) with teleosts having duplicates of some of these myosins (myo5aa, myo5ab, myo5ba, and $m y o 5 b b$ ), but not myo5c. A study of the duplicated genes in teleosts may provide insight into the evolutionary history of these genes and their linkage with specific cellular functions, namely transcriptional processing or splicing.

\section{Materials and methods}

RNA and cDNA isolation, RT-PCR and phylogenetic analyses were carried out as described in Nuckels et al. 2011 [1].

\section{Results}

An alignment of myo5a, myo5b, and myo5c revealed the conserved regions of the duplicated genes of teleosts. The $5^{\prime}$ myosin head (motor domain) and the 3' tail (cargo binding) are both highly conserved across taxa and between duplicates, reflecting the importance of these domains in light of 300 million plus years of evolutionary selection. The long neck region of the myo5 genes is less conserved and contributes to a more robust phylogenetic analysis. Using RT-PCR and qPCR we amplified fragments of myo5aa, myo5ab and myo5c from zebrafish tissues isolated at several developmental time points. This approach revealed differential expression between these duplicates with myo5aa being expressed at all developmental time points in whole zebrafish. myo5aa is also expressed in whole eyes whereas myo5ab seems to be weakly expressed or not expressed at all, depending on the developmental time point.

\section{Conclusions}

Phylogenetic analysis reveals that different regions of the myo5 genes evolve at different rates. As expected, exons encoding the ATP-binding and motor domains are slowly evolving relative to regions of the gene not involved in a motor or functional domain. Additional studies of expression may reveal subfunctionalization with one duplicate involved in RNA splicing and the other involved in organelle movements, further highlighting the benefit of using a teleost model to study evolutionary consequences of gene duplication.

\section{Acknowledgements}

We thank Dr. Jeff Gross (UT-Austin) for reagents and access to zebrafish. We thank Dr. Chris Nice (Texas State) for insightful comments. RN is supported by NSF GK12 grant Project Flowing Waters to Drs. Julie Westerlund, Tim Bonner, Weston Nowlin and Richard Earl of Texas State University, San Marcos.

Published: 8 April 2013

\section{References}

1. Nuckels RJ, Forstner MRJ, Capalbo-Pitts EL, Garcia DM: Developmental expression of muscarinic receptors in the eyes of zebrafish. Brain Research 2011, 1405:85-94.

2. de Lanerolle P, Serebryannyy L: Nuclear actin and myosins: Life without filaments. Nature Cell Biology 2011, 13:1282-1288.

\section{doi:10.1186/1756-8935-6-S1-P119}

Cite this article as: Nuckels and Garcia: Myosin 5 evolution and expression in teleosts. Epigentics \& Chromatin 2013 6(Suppl 1):P119. 